Fine mapping of a swine quantitative trait locus for number of vertebrae and analysis of an orphan nuclear receptor, germ cell nuclear factor (NR6Al)

\author{
Satoshi Mikawa, ${ }^{1,4}$ Takeya Morozumi, ${ }^{2}$ Shin-Ichi Shimanuki, ${ }^{2,3}$ Takeshi Hayashi, ${ }^{1}$ \\ Hirohide Uenishi, ${ }^{1}$ Michiko Domukai, ${ }^{2}$ Naohiko Okumura, ${ }^{2}$ and Takashi Awata ${ }^{1}$ \\ ${ }^{1}$ Division of Animal Sciences, National Institute of Agrobiological Sciences, Tsukuba, Ibaraki 305-8602, Japan; ${ }^{2}$ Second Research \\ Division, STAFF-Institute, Tsukuba, Ibaraki 305-0854, Japan
}

\begin{abstract}
The number of vertebrae in pigs varies and is associated with meat productivity. Wild boars, which are ancestors of domestic pigs, have 19 vertebrae. In comparison, European commercial breeds have 21-23 vertebrae, probably owing to selective breeding for enlargement of body size. We previously identified two quantitative trait loci (QTL) for the number of vertebrae on Sus scrofa chromosomes (SSC) 1 and 7. These QTL explained an increase of more than two vertebrae. Here, we performed a map-based study to define the QTL region on SSCl. By using three $\mathrm{F}_{2}$ experimental families, we performed interval mapping and recombination analyses and defined the QTL within a 1.9-cM interval. Then we analyzed the linkage disequilibrium of microsatellite markers in this interval and found that 10 adjacent markers in a 300-kb region were almost fixed in European commercial breeds. Genetic variation of the markers was observed in Asian local breeds or wild boars. This region encoded an orphan nuclear receptor, germ cell nuclear factor (NR6Al, formerly known as GCNF), which contained an amino acid substitution (Prol92Leu) coincident with the QTL. This substitution altered the binding activity of NR6Al to its corepressors, nuclear receptor-associated protein 80 (RAP80) and nuclear receptor corepressor 1 (NCORI). In addition, somites of mouse embryos demonstrated expression of NR6A1 protein. Together, these results suggest that NR6Al is a strong candidate for one of the QTL that influence number of vertebrae in pigs.
\end{abstract}

[Supplemental material is available online at www.genome.org. The sequence data from this study have been submitted to EMBL/GenBank/DDB] under accession nos. AB248749-AB248751, AP009124; accession nos. for STS are given in Supplemental Table 1].

Wild boars, which are the ancestors of modern domestic pigs, uniformly have 19 vertebrae. In comparison, European commercial breeds have 21-23 vertebrae (King and Roberts 1960). These breeds have long been selectively bred for enlargement of body size to increase meat production and improve reproductive performance. This process presumably increased the number of vertebrae. In mammals, the vertebral formula shows developmental constraint (Narita and Kuratani 2005). The number of cervical vertebrae is fixed at seven, and the total number of thoracic and lumbar vertebrae tends to be 19 , although the respective counts vary among species. For example, in the Monotremata, Marsupialia, Lagomorpha, Rodentia, and Artiodactyla, the total number of thoracic and lumbar vertebrae is conserved at 19 , which is thought to be the primitive form. In comparison, this number is increased in the Perissodactyla (e.g., horse, 24 vertebrae) and Carnivora (e.g., dog, 20 vertebrae) and that of the Primata is decreased to 17 . However, these changes are lineage specific, and variation is restricted in each species, as is seen in the Primata (Pilbeam 2004). In light of these findings, it is interesting that the

\footnotetext{
${ }^{3}$ Present address: Maebashi Institute of Animal Science, Maebashi, Gunma 371-0121, Japan.

${ }^{4}$ Corresponding author.

E-mail mikawa@affrc.go.jp; fax +81-29-838-8610.

Article published online before print. Article and publication date are at http:// www.genome.org/cgi/doi/10.1101/gr.6085507.
}

number of vertebrae in pigs varies from 19 to 23 within a single species.

In previous papers, we reported two quantitative trait loci (QTL) affecting the number of vertebrae on Sus scrofa chromosomes (SSC) 1 and 7; these QTL were identified using nine $\mathrm{F}_{2}$ families produced by crossing between breeds of European, Asian, and miniature pigs (Wada et al. 2000; Mikawa et al. 2005). These two QTL acted independently without an epistatic effect, and each had mainly an additive effect. For the QTL on SSC1, all the alleles of European commercial pigs used in the experimental families increased the number of vertebrae by $0.44-0.68$ per allele. For the QTL on SSC7, some European alleles similarly increased the number of vertebrae $(0.38-0.68)$. The combined effect of the two QTL accounted for an increase of more than two vertebrae. In $\mathrm{F}_{2}$ populations in which alternative alleles for both QTL were fixed in founder breed pigs, the proportions of phenotypic variance in the number of vertebrae explained by the QTL on SSC1 and SSC7 were similar, at $\sim 30 \%$ (Mikawa et al. 2005). In the current report, we describe our map-based study of the QTL on SSC1 and present a 300-kb region that is almost fixed in a variety of European commercial breeds. We also suggest that an orphan nuclear receptor, germ cell nuclear factor (NR6A1, formerly known as GCNF) is a strong candidate for the gene underlying this QTL, in light of analyses of polymorphism, function, and expression. 


\section{Results}

\section{Defining the QTL region by using $\mathrm{F}_{2}$ families}

In our previous studies, we mapped a QTL for the number of vertebrae on SSC1qter, between microsatellite markers SW1957 $(151.6 \mathrm{cM})$ and $S W 1301(175.8 \mathrm{cM})$ (Wada et al. 2000; Mikawa et al. 2005). To restrict the candidate region, three families in the previous study were reanalyzed by using microsatellite markers distributed densely throughout the QTL region (Mikawa et al. 2004). First, we performed an interval mapping using a subfamily of a Large White $\times$ Japanese wild boar population, for which construction three Large White female pigs (W1, W2, and W3) were used as parents. In the previous study, we reported that in two of them (W2 and W3) the QTL on SSC7 had no effects on number of vertebrae. In the subfamily derived from W2 and W3, which consisted of $207 \mathrm{~F}_{2}$ animals, the effect of increasing the number of vertebrae was attributable only to SSC1, and the proportion of phenotypic variance explained by the QTL on SSC1 was $\sim 60 \%$. As a result of interval mapping, the peak $F$-ratio (187.2) was detected between SJ344 (160.5 cM) and SJ861 (162.2 cM) (Fig. 1A). To evaluate the effect of sampling error on the estimated QTL position, we constructed a 95\% confidence interval for the position showing the highest $F$-ratio, with 1000 repetitions of bootstrap sampling. We obtained the region from 159.5 to $163.6 \mathrm{cM}$ as the $95 \%$ confidence interval for the QTL position (Fig. 1A).

Another interval mapping was performed using a Chinese Jinhua $\times$ Duroc population, which was the largest population in our studies and consisted of $549 \mathrm{~F}_{2}$ animals; in this population, alternative alleles were fixed in founder breed pigs in the QTLs on both SSC1 and SSC7. When the genotype of a marker near the QTL detected on SSC7 was included as a covariate in the model for the analysis of SSC1, the peak F-ratio (171.2) was obtained at $S J 344(160.5 \mathrm{cM})$ on SSC1 (Fig. 1A). A bootstrap analysis (with 1000 repetitions) showed that the 95\% confidence interval ranged from 159.8 to $161.4 \mathrm{cM}$ (Fig. 1A). We think that the results of these two independent analyses provide sufficient evidence for us to deduce that the QTL was located in the region from 159.5 to $163.6 \mathrm{cM}$.

The third $\mathrm{F}_{2}$ family was produced by crossing Chinese Meishan females with a Göttingen miniature male. In this population, a QTL for vertebral number was detected on SSC1 but not on SSC7. QTL analysis showed that the Göttingen miniature sire was heterozygous on SSC1: one allele (Ver) had the effect of increasing vertebral number, whereas

A the other had no effect and was thought to be wild type (wt). The Meishan dams were homozygous wild type (wt/wt) (Mikawa et al. 2005). Among the eight $F_{1}$ dams, five were homozygous wild type (wt/wt) and two were heterozygous (Ver/wt), but the genotype of the remaining animal was uncertain because of recombination within the QTL region that originated from the Göttingen miniature chromosome. By using microsatellite markers, we mapped the recombination site between $S J 344(160.5 \mathrm{cM})$ and SJ270 $(161.1 \mathrm{cM})$. The genomic region proximal to SJ344 was from the Ver-containing chromosomal segment from the sire (Fig. 1B). The heterozygosity of the QTL of the $\mathrm{F}_{1}$ dam with the recombination was suggested by the segregation of the number of vertebrae in $18 \mathrm{~F}_{2}$ animals produced from the $\mathrm{F}_{1}$ dam. This segregation was confirmed through correction for the QTL effects from the $\mathrm{F}_{1}$ sire $(\mathrm{Ver} / \mathrm{wt})$ when we assumed that the Ver allele increased the number of vertebrae by 0.57 , the average of the QTL effects in the experimental families in our previous study. We therefore judged that the QTL was located proximal to SJ270,

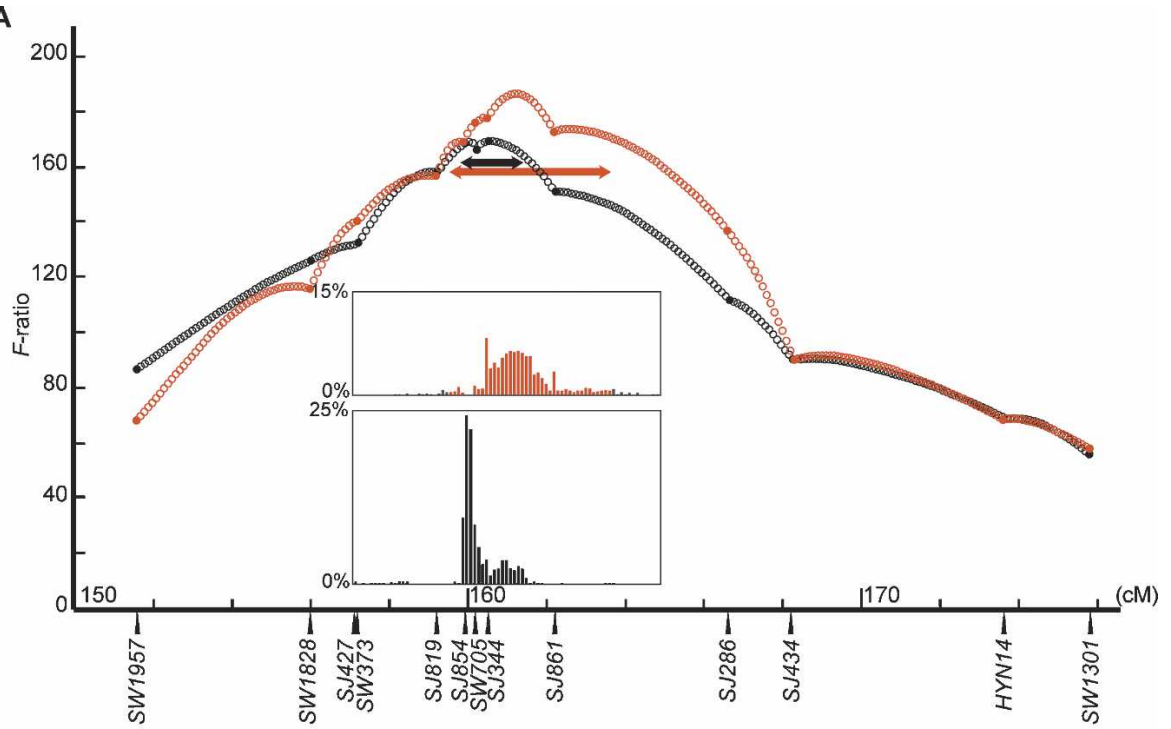

B $F_{1}$ dam with chromosomal recombination in QTL region

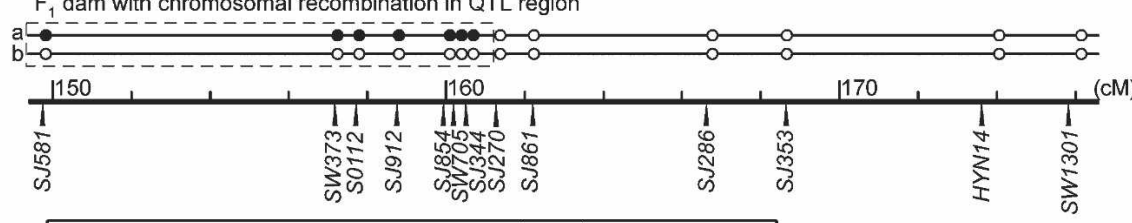

Average number of vertebrae in $F_{2}$ progeny $( \pm S E)$

a; $20.50 \pm 0.27(n=8) \quad$ b; $19.70 \pm 0.21(n=10) \quad p=0.015$

Corrected with QTL effect from $F_{1}$ sire $(V e r=w t+0.57)$

a; $20.14 \pm 0.23(n=8) \quad b ; 19.53 \pm 0.17(n=10) \quad p=0.021$

Figure 1. Dissection of a QTL region on $\mathrm{SSC} 1$ for vertebral number in $\mathrm{F}_{2}$ families. ( $A$ ) Plots of $F$-ratio for interval mapping analyses in the Large White $\times$ Japanese wild boar population (red) and Jinhua $\times$ Duroc cross population (black). For the latter, the QTL effect on SSC7 was removed by incorporating the genotype of a marker in the QTL region into the model as a covariate. Bootstrap analysis (with 1000 repetitions) was performed to obtain the $95 \%$ confidence interval of the estimated QTL position on SSC1, and the results are shown in the inset bar graphs. Double-ended arrows indicate the $95 \%$ confidence interval in each population. (B) QTL type of an $\mathrm{F}_{1}$ dam with a recombination in the QTL region. The dam was from a Meishan $\times$ Göttingen miniature cross. Meishan pigs were wt/wt, and the Göttingen miniature was heterozygous (wt/Ver) at the QTL. Solid circles indicate alleles from the parental chromosome with the Ver alleles. Clear circles indicate alleles from the parental chromosomes with the wt allele. Average number of vertebrae was compared between two groups of $F_{2}$ animals produced from the $F_{1}$ dam, categorized according to transmitted chromosomes (indicated by $\mathrm{a}$ or $\mathrm{b})$. Correction for the QTL effect from the $\mathrm{F}_{1}$ sire (Ver/wt) was also used. We assumed that the Ver allele increased the number of vertebrae by 0.57 , an average effect in the experimental population in our previous study. (SE) Standard error. 
and the results suggested that the QTL was located between SJ819 $(159.2 \mathrm{cM})$ and SJ270 $(161.1 \mathrm{cM})$. To further define the QTL, we then constructed a BAC contig for this region and developed microsatellite markers (Supplemental Table 1; Fig. 2A-C).

\section{Genetic variation of microsatellite markers in the QTL region}

We attempted to fine-map the QTL, in accordance with the assumption that the genetic variation around this QTL would be reduced in commercial breed pigs because vertebral number is associated with body size, which has been a focus of selective pig breeding. To analyze the genetic variation, we collected 194 independent samples from five commercial breeds (Landrace, Large White, Yorkshire, Duroc, and Berkshire) and 40 samples from Asian local breeds (Meishan, Jinhua, and Japanese wild boar) as references. Using these samples, we genotyped 24 microsatellite markers in the 1.9-cM region between SJ819 and SJ270. The re- sults revealed that $S J 878$ and SJ885 were nonpolymorphic in commercial breeds and that SJ641, SJ884, and SJ820 showed dramatically reduced polymorphism: The frequencies of all major alleles were $>0.99$. At $S W 705$, located between $S J 878$ and SJ885, the frequency of the major allele was 0.93 and that of the second allele, which was two nucleotides smaller than the major allele, was 0.05 in commercial breeds (Fig. 2C; Table 1). In comparison, the genetic variation of these markers was maintained in the Asian breeds.

To define the region of reduced genetic variation, we isolated 11 novel microsatellite elements (underlined in Fig. 2D) by sequencing the $600-\mathrm{kb}$ region between S/854 and S/872. Reanalysis with these 11 markers, in addition to the 24 markers described earlier, revealed that lack of genetic variation in commercial breeds occurred at 10 adjacent markers located in a 300-kb region between SJ641 and SJ820 (Fig. 2D,E; Table 1).
A

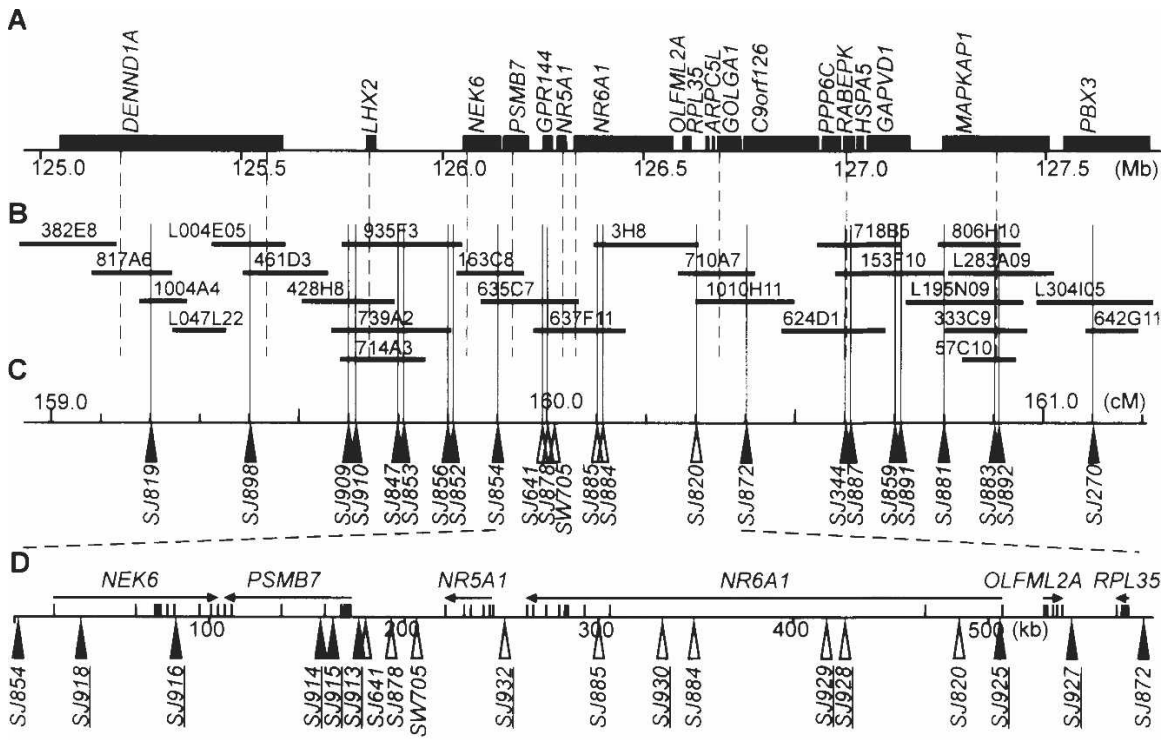

E

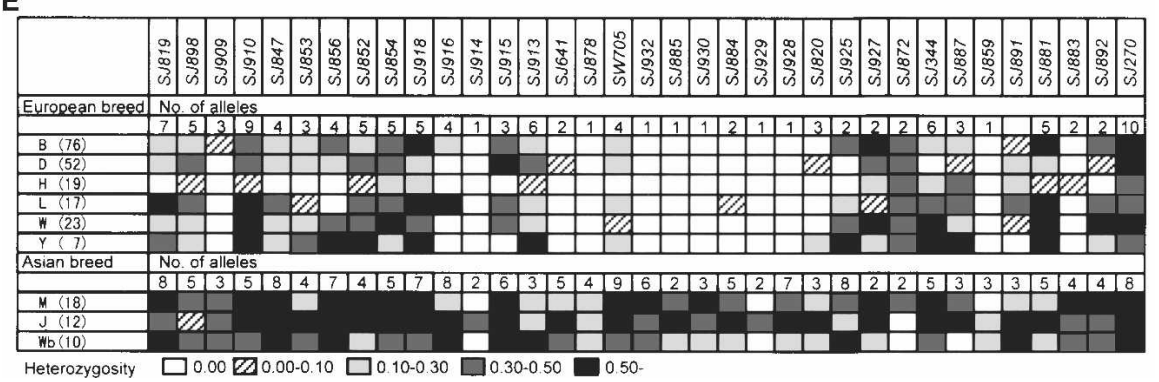

Figure 2. Analyses of genomic structure and genetic variation of the QTL region. $(A)$ Gene map of a part of the human chromosome 9qter. (B) BAC contig for the QTL region. Vertical lines indicate positions of STS (broken) and microsatellite markers (solid). BAC clones (Suzuki et al. 2000) from which markers were developed and those in the minimum tiling path are shown. (C) Linkage map of microsatellite markers on SSC1qter. Arrowheads indicate positions of microsatellite markers, and clear arrowheads indicate those lacking genetic variation in European commercial breed pigs. (D) Defining the QTL region by the genetic variation of microsatellite markers. Sequencing analysis was performed for the region from S/854 to S/872. Exons of the genes found in this region are indicated, and arrows show the directions of gene transcription. We isolated 11 novel markers (underlined) and added them to the analysis of genetic variation. A reduction of genetic variation of microsatellite markers in European commercial breed pigs occurred between S/641 and S/820. The GenBank accession number for the genomic sequence in this region is AP009124. (E) Heterozygosities of markers in each breed. Breeds are: B, Berkshire; D, Duroc; H, Hampshire; L, Landrace; W, Large White; Y, Yorkshire; M, Meishan; J, Jinhua; and Wb, Japanese wild boar; and numbers of samples are shown in parentheses.

\section{Candidate gene detection} and polymorphism analysis

The region from SJ641 to SJ820 contained two nuclear receptor genes: NR5A1 (formerly $A D 4 B P$; adrenal 4-binding protein) and NR6A1 (formerly GCNF; germ cell nuclear factor) (Fig. 2D). NR5A1 participates in gonadal differentiation and steroidogenesis (Luo et al. 1994; Shen et al. 1994). NR6A1 is an orphan receptor that is expressed in the testis and ovary (Katz et al. 1997; Zhang et al. 1998; Yang et al. 2003). NR6A1 also is expressed in early embryos (Lan et al. 2002, 2003), and Nr6a1-deficient mouse embryos display serious defects in somitogenesis, generating a maximum of 13 (instead of 25) somites (Chung et al. 2001). We sequenced the coding regions of NR5A1 and NR6A1 from 11 European and 14 Asian breed pigs used as parents in the $\mathrm{F}_{2}$ experimental families in our previous study (Mikawa et al. 2005) and identified only one amino acid substitution, coincident with the QTL in NR6A1 (Fig. 3A) but not in NR5A1. This substitution (Pro192Leu; $\mathrm{C} \rightarrow \mathrm{T}$ at nucleotide 748 of AB248749) was fixed as leucine in the 194 pigs of commercial breeds in the DNA panel. Asian pigs had proline, which is conserved in human and mouse NR6A1. In the region between the NR5A1 and NR6A1 genes or their introns, several single-nucleotide substitutions were fixed alternatively in European and Asian breeds (data not shown), but we could not evaluate their functional association with the QTL effect.

\section{Interaction of swine NR6Al and corepressors}

The amino acid substitution in NR6A1 was located in the hinge region between the DNA binding domain and the puta-

\section{Genome Research}

www.genome.org 
Downloaded from genome.cshlp.org on April 26, 2023 - Published by Cold Spring Harbor Laboratory Press

QTL for pig vertebral number encodes NR6A1

Table 1. Genetic variations of microsatellite markers in the QTL candidate region on SSC1 for number of vertebrae

\begin{tabular}{|c|c|c|c|c|c|c|c|c|c|c|}
\hline \multirow{3}{*}{$\begin{array}{l}\begin{array}{l}\text { Micro- } \\
\text { satellite } \\
\text { marker }\end{array} \\
\text { S/819 }\end{array}$} & \multicolumn{5}{|c|}{ European commercial breed pigs $(n=194)$} & \multicolumn{5}{|c|}{ Asian local breed pigs $(n=40)$} \\
\hline & \multirow{2}{*}{$\frac{\text { No. of alleles }}{7}$} & \multicolumn{4}{|c|}{ Ratio (fragment size) of top 4 alleles } & \multirow{2}{*}{$\frac{\text { No. of alleles }}{8}$} & \multicolumn{4}{|c|}{ Ratio (fragment size) of top 4 alleles } \\
\hline & & $0.86(258)$ & $0.07(262)$ & $0.03(264)$ & $0.02(268)$ & & $0.39(268)$ & $0.27(250)$ & $0.09(266)$ & $0.07(270)$ \\
\hline $5 / 898$ & 5 & $0.82(278)$ & $0.10(287)$ & $0.04(281)$ & $0.02(274)$ & 5 & $0.48(276)$ & $0.25(278)$ & $0.21(272)$ & 0.04 \\
\hline $5 / 909$ & 3 & $0.99(272)$ & $<0.01(290)$ & $<0.01(269)$ & - & 3 & $0.48(269)$ & $0.37(276)$ & $0.15(272)$ & - \\
\hline S/910 & 9 & $0.42(269)$ & $0.24(261)$ & $0.19(263)$ & $0.07(275)$ & 5 & $0.43(271)$ & $0.28(263)$ & $0.19(269)$ & 0.09 \\
\hline $5 / 847$ & 4 & $0.80(173)$ & $0.19(175)$ & $<0.01$ & $<0.01(177)$ & 8 & $0.27(175)$ & $0.23(179)$ & $0.15(173)$ & 0.13 \\
\hline S/853 & 3 & $0.93(156)$ & $0.06(158)$ & $0.01(162)$ & - & 4 & $0.70(156)$ & $0.24(160)$ & $0.03(163)$ & $0.03(177)$ \\
\hline $5 / 856$ & 4 & $0.65(200)$ & $0.19(202)$ & $0.15(206)$ & $0.01(208)$ & 7 & $0.27(200)$ & $0.25(206)$ & $0.23(192)$ & $0.09(202)$ \\
\hline S/852 & 5 & 0.45 (187) & $0.38(176)$ & $0.16(193)$ & $<0.01(181)$ & 4 & 0.70 (179) & $0.22(176)$ & $0.06(193)$ & $0.02(181)$ \\
\hline S/854 & 5 & $0.55(295)$ & $0.32(293)$ & $0.08(283)$ & $0.04(281)$ & 5 & $0.47(283)$ & $0.21(281)$ & $0.12(303)$ & 0.10 \\
\hline S/918 & 5 & $0.54(329)$ & $0.36(352)$ & 0.09 (354) & $<0.01(335)$ & 7 & $0.42(329)$ & $0.16(333)$ & $0.14(350)$ & $0.12(352)$ \\
\hline S/916 & 4 & $0.95(148)$ & $0.02(144)$ & $0.02(150)$ & $0.01(152)$ & 8 & $0.42(148)$ & $0.15(154)$ & $0.13(163)$ & 0.08 \\
\hline S/914 & 1 & $1.00(224)$ & - & - & - & 2 & $0.93(224)$ & $0.07(219)$ & - & - \\
\hline S/915 & 3 & $0.60(240)$ & $0.34(238)$ & $0.06(236)$ & - & 6 & $0.24(240)$ & $0.20(215)$ & $0.18(234)$ & $0.16(232)$ \\
\hline S/913 & 6 & $0.86(317)$ & $0.06(315)$ & $0.03(321)$ & $0.02(319)$ & 3 & $0.62(315)$ & $0.20(317)$ & $0.18(327)$ & - \\
\hline S) 641 & 2 & $0.99(300)$ & $0.01(302)$ & - & - & 5 & $0.50(296)$ & $0.16(302)$ & $0.16(300)$ & 0.13 \\
\hline $5 / 878$ & 1 & $1.00(264)$ & - & - & - & 4 & $0.40(284)$ & $0.28(272)$ & $0.16(278)$ & $0.16(264)$ \\
\hline SW705 & 4 & $0.93(146)$ & $0.05(148)$ & $0.01(144)$ & $0.01(142)$ & 9 & $0.25(157)$ & $0.20(159)$ & 0.15 (148) & $0.13(153)$ \\
\hline S/932 & 1 & $1.00(324)$ & - & - & - & 6 & $0.33(322)$ & $0.19(324)$ & $0.19(320)$ & 0.13 \\
\hline S/885 & 1 & $1.00(252)$ & - & - & - & 2 & $0.70(252)$ & $0.30(256)$ & - & - \\
\hline S/930 & 1 & $1.00(359)$ & - & - & - & 3 & 0.65 (359) & $0.33(357)$ & $0.02(361)$ & - \\
\hline S/884 & 2 & $1.00(332)$ & $<0.01(330)$ & - & - & 5 & $0.47(326)$ & $0.22(322)$ & 0.18 (328) & 0.07 \\
\hline S/929 & 1 & $1.00(326)$ & - & - & - & 2 & $0.93(326)$ & $0.07(330)$ & - & - \\
\hline S/928 & 1 & $1.00(218)$ & - & - & - & 7 & $0.30(222)$ & $0.27(218)$ & $0.18(226)$ & $0.13(216)$ \\
\hline $5 / 820$ & 3 & $0.99(165)$ & $<0.01(163)$ & $<0.01(171)$ & - & 3 & $0.41(165)$ & $0.39(163)$ & $0.20(168)$ & - \\
\hline S/925 & 2 & $0.78(381)$ & $0.22(374)$ & - & - & 8 & $0.57(268)$ & $0.13(374)$ & $0.12(273)$ & 0.05 \\
\hline S/927 & 2 & $0.65(275)$ & $0.35(273)$ & - & - & 2 & $0.67(271)$ & $0.33(273)$ & - & - \\
\hline S/872 & 2 & $0.55(233)$ & $0.45(229)$ & - & - & 2 & $0.90(229)$ & $0.10(233)$ & - & - \\
\hline S/344 & 6 & 0.71 (157) & $0.10(148)$ & 0.08 (159) & $0.06(161)$ & 5 & $0.44(148)$ & $0.43(157)$ & 0.09 (159) & $0.02(164)$ \\
\hline $5 / 887$ & 3 & $0.85(291)$ & 0.09 (299) & $0.06(301)$ & - & 3 & $0.83(276)$ & $0.10(299)$ & $0.07(291)$ & - \\
\hline $5 / 859$ & 1 & $1.00(333)$ & - & - & - & 3 & $0.76(333)$ & 0.21 (331) & $0.03(334)$ & - \\
\hline S/891 & 4 & $0.88(276)$ & $0.11(275)$ & $<0.01(277)$ & $<0.01(280)$ & 3 & $0.79(275)$ & $0.18(266)$ & $0.03(276)$ & - \\
\hline S/881 & 5 & $0.39(216)$ & $0.30(210)$ & $0.26(204)$ & $0.04(218)$ & 5 & $0.52(210)$ & $0.26(212)$ & $0.15(214)$ & $0.06(216)$ \\
\hline $5 / 883$ & 2 & $1.00(255)$ & $<0.01(247)$ & - & - & 4 & 0.39 (249) & $0.37(253)$ & $0.22(251)$ & $0.02(247)$ \\
\hline S/892 & 2 & $0.62(199)$ & $0.38(201)$ & - & - & 4 & $0.37(197)$ & 0.35 (199) & $0.16(201)$ & $0.12(195)$ \\
\hline S/270 & 10 & $0.37(171)$ & $0.34(179)$ & $0.10(165)$ & $0.06(183)$ & 8 & 0.21 (185) & 0.16 (187) & $0.16(181)$ & $0.16(177)$ \\
\hline
\end{tabular}

tive ligand-binding domain. The hinge domain of NR6A1 is reported to be essential for its interaction with the corepressors, nuclear receptor corepressor 1 (NCOR1) and nuclear receptorassociated protein 80 (RAP80) (Yan et al. 2002). To analyze the influence of Pro192Leu on the binding of NR6A1 to its corepressors we used two-hybrid systems. The pig leucine-type NR6A1 showed three times higher binding activity to pig RAP80 than did the proline-type protein (Fig. 3B). For NCOR1, the binding activity of the leucine form of NR6A1 was twice as high as that of the proline form when an NCOR1 C-terminal peptide (454 amino acid residues) containing the interacting domains (ID-I and ID-II [Yan and Jetten 2000]) was used (Fig. 3B).

\section{Expression of Nrbal in mouse embryos}

We analyzed the expression of Nr6a1 in mouse embryos (embryonic day [ED] 10.5). By using in situ hybridization, we detected only very faint signals for Nr6a1 mRNA in three tissuesthe mandibular component of the first branchial arch, the lung bud, and the somites (Fig. 4), while its corepressors' (Rxrip110, mouse homolog of RAP80, and Ncor1) mRNA were detected in a variety of tissues (Supplemental Fig. 1A,B). While the expression of Nr6a1 mRNA was so faint and hardly detectable, somites on both sides of the notochord were intensely immunostained for Nr6a1 protein (Fig. 5). Because of the many molecular mechanisms conserved in the embryonic development of mammals, we expect that the expression patterns of pig NR6A1 and its corepressors would be similar to those we noted in mice.

\section{Discussion}

Domestic pigs in Europe have been under consistent selective breeding since the 19th century. European commercial breed pigs now grow faster and larger than Asian breeds or wild boars. The increase in vertebral number is a factor in these improvements. We suspected that selective breeding had reduced the genetic variation around the responsible locus, but we had not expected that fixation around the QTL for the number of vertebrae would affect such a wide (300-kb) region throughout a variety of commercial breeds. This result suggests that this QTL was the result of a single allele mutation rather than polymorphism and that the effect of the allele was very important for pig breeding. It is difficult to evaluate when and how the fixation occurred but it would occur at least before the establishment of present European commercial breeds (Large White, Yorkshire, Berkshire, Duroc, Hampshire, Landrace, etc.).

NR5A1 and NR6A1 are located in this 300-kb region. NR5A1 participates in gonadal differentiation and steroidogenesis and is expressed in primary steroidogenic tissues (Luo et al. 1994; Shen et al. 1994). Despite normal survival in utero, all Nr5a1-null mice die by postnatal day 8 , and these animals lack adrenal glands and gonads and are severely deficient in corticosterone (Luo et al. 
A Pig (wt) 172 HGDSDHSSPGNRASESNQPSPGSTLSS-RSVELNGFMAFRD

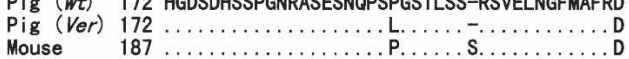

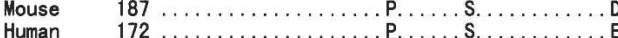
Pig (wt) 212 QYMGMSVPPHYQYIPHLFSYSAHSPLLPPQARSLDPQSYSL

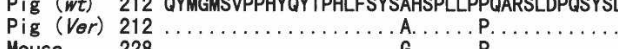

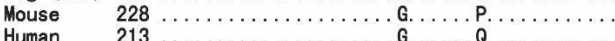

B
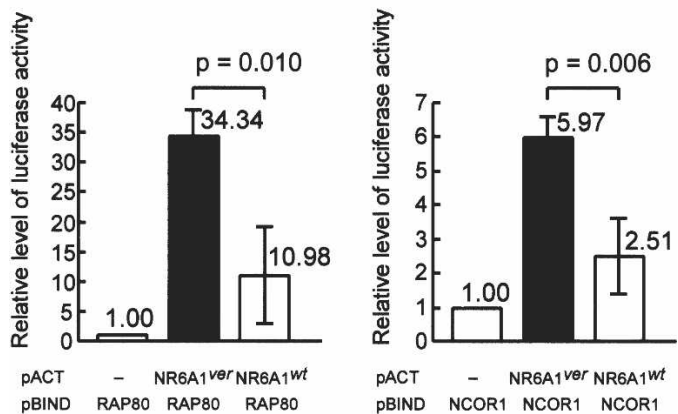

Figure 3. (A) Comparison of amino acid sequences of Nr6a1 hinge domains of pig, human, and mouse. We identified an amino acid substitution (Pro192Leu; C $\rightarrow$ T at nucleotide 748 of AB248749) in pig NR6A1. Leucine occurred in alleles increasing vertebral number (Ver), whereas proline occurred in the wild-type pig allele (wt) as well as in human and mouse NR6A1/Nr6a1. (B) Effect of an amino acid substitution in pig Nr6a1 on its interaction with RAP80 and NCOR1. Interaction of pig NR6A1 and its corepressors RAP80 and NCOR1 was analyzed with a two-hybrid system in mammalian cells. $\mathrm{CHO}$ cells were transfected with pACT and pBIND plasmids containing the indicated DNA fragments. After $48 \mathrm{~h}$, cells were collected and assayed for reporter activity. Relative luciferase activity was plotted and compared with the results of $t$-tests; error bars, 1 SD.

1994). Most of the in vivo function of NR6A1 is still unknown. Nr6a1 is expressed in the testis, ovary, and early embryos, and Nr6a1-deficient mouse embryos cannot survive beyond ED10.5, owing to cardiovascular defects and failure to establish an appropriate chorioallantoic connection. In addition, mutant embryos display serious defects in somitogenesis, generating a maximum of 13 (instead of 25) somites (Chung et al. 2001), although it is possible that malformation of somites was caused by the termination of other biological processes independent of somitogenesis. In Xenopus, NR6A1 may play a role in the formation of the anterior-posterior axis (David et al. 1998). In the human genome, a predicted gene, GPR144 (G protein-coupled receptor 144 ), is located proximal to NR5A1. We found that the pig genome contains GPR144-homologous sequences but not those corresponding to exons 1 and 20, where the start and stop codons, respectively, are located in the human homolog. Furthermore, in the pig sequences we found stop codons in the frame corresponding to the open reading frame of human GPR144. We therefore think that GPR144 is nonfunctional in pigs.

We identified an amino acid substitution (Pro192Leu) in the hinge region of NR6A1, and this substitution is coincident with the QTL. Nuclear receptors are reported to bind coregulators and function as repressors or activators. For example, retinoic-acid and thyroid-hormone receptors bind NCOR1 (Horlein et al. 1995) or NCOR2 (SMRT [silencing mediator of retinoic acid and thyroid hormone receptors]) (Chen and Evans 1995) as corepressors. Yan and Jetten (2000) reported that NR6A1 binds NCOR1 but not NCOR2; they also isolated a novel nuclear protein, RAP80, which interacts with NR6A1 as a corepressor. The hinge domain of NR6A1 is essential for its interaction with NCOR1 and
RAP80 (Yan et al. 2002). The amino acid substitution (Pro182Leu) in the hinge regions altered the binding activity of NR6A1 to NCOR1 and RAP80, although the biological significance of this effect is unknown.

These findings, as well as our evaluation of Nr6a1 expression in the somites of ED10.5 mouse embryos, suggested that NR6A1 was a strong candidate for the QTL. Because our map-based study excluded the promoter region of NR6A1 and because Pro192Leu was a functional polymorphism, this mutation may be the cause of the QTL. However, this hypothesis cannot exclude the possibility of single-nucleotide polymorphisms (such as those in introns or unknown genes including those for noncoding RNAs) that alter the regulation of expression of the QTL.

In pigs as livestock, meat productivity is associated with vertebral number; therefore isolation of the QTL for vertebral number is valuable. The information we present likely will be useful in breeding and construction of new pig lines, especially when Asian pigs are used as genetic resources. Furthermore, we propose a putative functional role for NR6A1 in somitogenesis. Vertebral morphology is associated with the expression pattern of Hox genes (Burke et al. 1995). Disruption of Gdf11, Mll, and Bmi1, which are located upstream of the Hox genes, shifts the expression boundaries of the Hox genes and alters vertebral morphology (Yu et al. 1995; Hanson et al. 1999; McPherron et al. 1999; Dubrulle et al. 2001). The "segmentation clock" is also essential in directing vertebral morphology and is closely linked to the Notch and Wnt signaling pathways (Cordes et al. 2004). The mouse mutation vestigial tail $(v t)$ shows reduced transcription of Wnt $3 a$ and altered vertebral morphology (Greco et al. 1996). We think it will be important to investigate the involvement of NR6A1 in Hox expression patterning and regulation of the segmentation clock.

Recently, some progress has been made in understanding the targets of NR6A1 in the developmental process of early embryos. Sato et al. (2006) reported that NR6A1 binds the DR0 element (Chen et al. 1994) in the promoter region of Oct3/4 and recruits DNA methyltransferase for Oct3/4 silencing. OCT3/4 is required for early embryonic cells to maintain pluripotency $(\mathrm{Ni}-$ chols et al. 1998) and also plays a crucial role in the specification of the first embryonic lineage (Niwa et al. 2000). Hentschke et al.
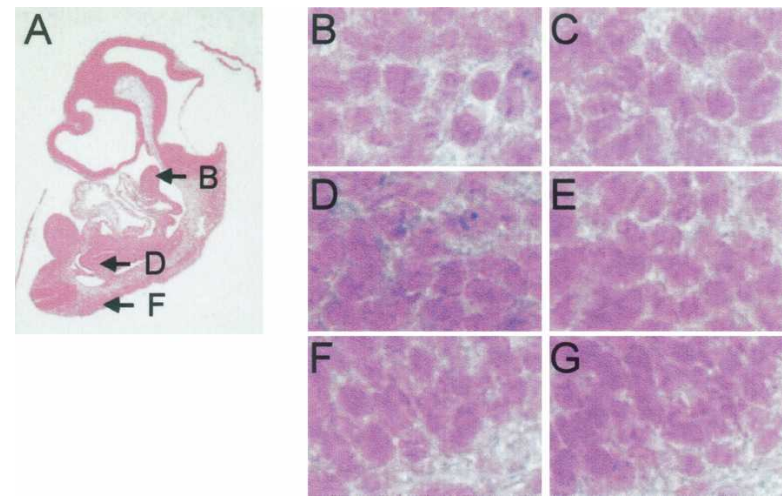

Figure 4. Expression of Nr6a1 mRNA in embryonic day (ED)10.5 mouse embryos. Sense $(A, B, D, F)$ and antisense $(C, E, G)$ probe for Nr6a1 mRNA were hybridized to sections of ED10.5 mouse embryos. Faint signals for Nr6a1 mRNA were detected with antisense probe in the mandibular component of the first branchial arch $(B)$, lung bud $(D)$, and somites $(F)$. No signals were detected in these tissues with sense probe $(C, E, G)$. Lines with characters in the left image $(A)$ indicate the positions of tissues in which signals were detected. 
A

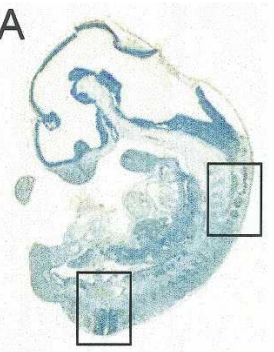

F

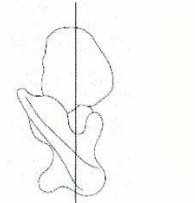

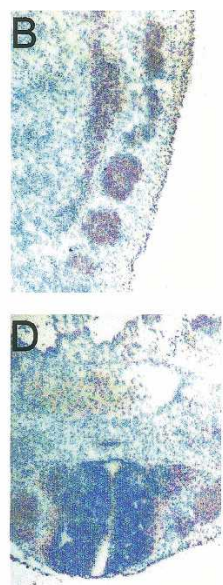

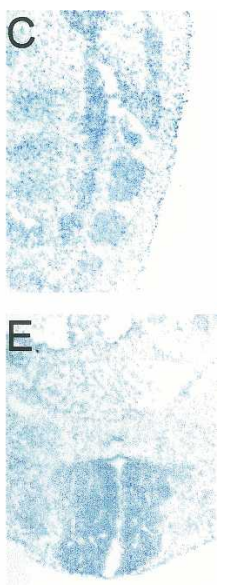

Figure 5. Immunohistochemical study of Nr6a1 protein in embryonic day (ED)10.5 mouse embryos. Frozen sections of ED10.5 mouse embryos were fixed by ethanol and incubated either with rabbit anti-Nr6a1 antibodies $(A, B, D)$ or with rabbit IgG isolated from preimmune sera $(C, E)$. Signals were present in somites on both sides of the notochord. The vertical line in the sketch $(F)$ indicates the location of the sections, and the photomicrographs at right $(B, D)$ are magnified images of the boxed regions at left $(A)$.

(2006) reported that NR6A1 is a repressor of CRIPTO, a coreceptor for the morphogen nodal. NR6A1-mediated repression of the CRIPTO promoter is also dependent upon the DR0 site. CRIPTO is required for correct orientation of the anterior-posterior axis in the mouse embryo (Ding et al. 1998). NR6A1 was originally identified as the GCNF expressed predominantly in germ line, but now its importance in embryonic development has been realized. We think that these findings support the idea that NR6A1 is a strong candidate of the QTL for the number of vertebrae.

\section{Methods}

\section{QTL scanning for number of vertebrae on SSCl}

A QTL scan on SSC1 was performed for the number of vertebrae in both the Large White $\times$ Japanese wild boar population and the Jinhua $\times$ Duroc cross population. An interval mapping based on the least-squares method developed for outbred population (Haley et al. 1994) was used, whereby a QTL was scanned every $0.1 \mathrm{cM}$ on SSC1. At a QTL, we assumed that the grandparental breeds were fixed for alternative alleles, $\mathrm{Q}$ and $\mathrm{q}$. Denoting the effect of QQ as $a$, the effect of Qq as $d$, and the effect of qq as $-a$, the phenotypic value of the $i$ th $\mathrm{F}_{2}$ individual, $y_{i}$, can be expressed as the following linear model:

$$
y_{i}=\mu+u_{i} a+v_{i} d+e_{i}
$$

where $\mu$ is the intercept of the model; $u_{i}$ is the coefficient for the additive effect of a putative QTL for the $i$ th individual, which [denoting the probability of an individual being genotype $\mathrm{AB}$ as $\operatorname{Pr}(\mathrm{AB})]$ is given as $\operatorname{Pr}(\mathrm{QQ})-\operatorname{Pr}(\mathrm{qq})$; $v_{i}$ is the coefficient for the dominance effect of a putative QTL, which is equal to $\operatorname{Pr}(\mathrm{Qq})$; and $e_{i}$ is the residual error. This model was fitted in the analysis of a QTL on SSC1 for a Large White $\times$ Japanese wild boar population in which only the QTL on SSC1 was found to segregate in our previous study. For another $\mathrm{F}_{2}$ population, i.e., a Jinhua $\times$ Duroc population in which two QTL had been detected, on SSC1 and SSC7, in our previous study, analysis of the QTL on SSC1 was performed using the modified model, which

incorporated the genotype of a microsatellite marker (SW252) nearest to the QTL position on SSC7 as a covariate. The modified model can be written as

$$
y_{i}=\mu+u_{i} a+v_{i} d+w_{i} b+e_{i},
$$

where $w_{i}$ is the coefficient for the additive effect of a QTL on SSC7, which is given as $\operatorname{Pr}(\mathrm{QQ})-\operatorname{Pr}(\mathrm{qq})$ assuming that the QTL is located close to SW252; and $b$ is the additive effect of the QTL. The results of our previous analysis suggested that the QTL on SSC7 was additive; therefore, we considered only the additive effect of the QTL.

For both populations, 95\% confidence intervals for the QTL on SSC1 were obtained by bootstrap analysis of 1000 repetitions (Visscher et al. 1996). In these analyses, the map positions of our linkage map (Mikawa et al. 2004) were used.

\section{Isolation of bacterial artificial chromosomes (BAC) clones and development of microsatellite markers}

The QTL region of SSC1qter corresponds to a region of the human chromosome 9ter (Mikawa et al. 2004). Swine sequencetagged sites (STSs) were developed from swine expressed sequence tags (ESTs) or swine whole-genome shotgun sequences, which were obtained by BLAST searches with the human gene sequences on the chromosome 9 and then confirmed to be the targeted sequences by using BLAST searches inversely against the human genome (Supplemental Table 1). BAC clones (Suzuki et al. 2000) were isolated with swine STS and BAC end sequences were used for chromosome walking. Microsatellite sequences were isolated from BAC clones by using a direct sequencing method reported previously (Fujishima-Kanaya et al. 2003). Genotyping of microsatellite markers was performed with the ABI Prism 3700 DNA Analyzer and GeneScan analysis software (Applied Biosystems).

\section{Swine genomic DNA panel}

For analysis of genetic variation in pigs, a DNA panel was constructed from 194 samples of European commercial-breed pigs and 40 samples of Asian local-breed pigs. For Berkshire samples $(n=76)$, DNA was prepared from samples of pork meat produced in Japan, the United States, and the United Kingdom. For samples from Duroc $(n=52)$, Landrace $(n=17)$, Large White $(n=23)$, and Yorkshire $(n=7)$ breeds, DNA was prepared from semen provided for artificial insemination in Japan. The donor male pigs were confirmed to be without consanguinity for three generations. For Hampshire breed samples $(n=19)$, DNA was prepared from animals bred at the experimental stations of Kumamoto and Okinawa prefectures in Japan. For Meishan samples $(n=18)$, DNA was prepared from animals bred at the National Institute of Livestock Breeding, Japan. For Jinhua samples $(n=12)$, DNA was prepared from animals bred at the Shizuoka Swine and Poultry Experiment Station in Japan. For Japanese wild boars, DNA was prepared from 10 animals from six different prefectures.

\section{Plasmid construction for two-hybrid analysis}

Swine RAP80 cDNA (AB248750) was cloned from testis cDNA by using the 5'RACE System (Invitrogen) and RT-PCR with primers designed from swine EST (BX923822, CF180155) homologous to human RAP80 cDNA. Swine NCOR1 partial cDNA (AB248751) containing ID-I and ID-II (Yan and Jetten 2000) was cloned using the 3'RACE System (Invitrogen) from the sequence of swine EST (BX915421, AU296541) homologous to human NCOR1. These DNA fragments were inserted into pBIND vector, in which the Renilla luciferase gene was located as an internal control (Check- 
Mate Mammalian Two-Hybrid System, Promega). Proline-type and leucine-type NR6A1 cDNA (AB248749) were cloned from testis cDNA by using RT-PCR. From the NR6A1 cDNA, DNA fragments spanning the region from the hinge domain to the $\mathrm{C}$ terminus (nucleotides 687-1613 of AB248749, equivalent to the $\Delta \mathrm{N} 1$ construct (Yan et al. 2002), were inserted into pACT vector (CheckMate Mammalian Two-Hybrid System, Promega).

\section{Mammalian two-hybrid analysis}

CHO cells $\left(2 \times 10^{5} /\right.$ well $)$ were plated in six-well dishes and $20 \mathrm{~h}$ later transfected in F12 medium with a reporter plasmid, pG5luc (Promega), in which the firefly luciferase gene was located, and with pACT and pBIND expression plasmids (each $0.5 \mu \mathrm{g}$ ) by using $3 \mu \mathrm{l}$ of FuGENE 6 transfection reagent (Roche Molecular Biochemicals). Cells were collected $48 \mathrm{~h}$ after transfection and assayed for both firefly and Renilla luciferases by using a Dual-Glo Luciferase Reporter Assay System (Promega). Renilla luciferase activity was used as an internal control to monitor transfection efficiency. Transfections were performed in triplicate, and each experiment was repeated three times.

\section{In situ hybridization of Nr6al and corepressors in mouse embryo}

In situ hybridization on mouse embryo (ED10.5) sections was performed as described previously (Hoshino et al. 1999). Mouse embryos were fixed in $4 \%$ paraformaldehyde and dehydrated in a step-wise manner with ethanol. Sections $(6 \mu \mathrm{m})$ were made from wax-embedded preparations. For Nr6a1 probes, 1586-2084 bp of NM_010264 was amplified from testis RNA by PCR primers with T7 or SP6 promoter sequences. For probes to Ncor1 and Rxrip110 (mouse homolog of RAP80), 2319-2739 bp of NM_011308 and 467-893 bp of NM_011307 were amplified by PCR, respectively, as well. Digoxygenin-labeled RNA probes were prepared by in vitro transcription with a DIG RNA Labeling Mix (Roche Molecular Biochemicals). Hybridization was performed with probes at concentrations of $200-500 \mathrm{ng} / \mathrm{mL}$ in hybridization solution ( $50 \%$ formamide, $5 \times$ SSC, $1 \%$ SDS, $50 \mu \mathrm{g} / \mathrm{mL}$ tRNA, and $50 \mu \mathrm{g} / \mathrm{mL}$ heparin) at $55^{\circ} \mathrm{C}$ for $16 \mathrm{~h}$. After hybridization, the specimens were washed, and this was followed by RNase treatment. After treatment with $0.5 \%$ blocking reagent (Roche Molecular Biochemicals) in TBST for $1 \mathrm{~h}$, the samples were incubated with anti-DIG AP conjugate (Roche Molecular Biochemicals), and staining reactions were performed with NBT/BCIP (Roche Molecular Biochemicals), followed by counterstaining with nuclear fast red (Sigma-Aldrich).

\section{Anti-NR6Al polyclonal antibodies and immunohistochemistry}

A NR6A1-specific polypeptide (amino acids 36-50, CQDELAELDPSTISV) was synthesized, purified by HPLC, conjugated to keyhole limpet hemocyanin, and then injected into rabbits to generate rabbit anti-NR6A1 antibodies. Preimmune and hyperimmune sera were collected and passed through HiTrap protein A HP affinity columns (Amersham) to generate rabbit preimmune immunoglobulin $\mathrm{G}$ (IgG) and rabbit anti-NR6A1 IgG, respectively. Frozen sections of mouse embryos (ED10.5) were fixed in ethanol at $4^{\circ} \mathrm{C}$ for $10 \mathrm{~min}$, dried, and washed with distilled water. Samples were incubated for $2 \mathrm{~h}$ either with the preimmune IgG or with purified anti-NR6A1 antibodies. After extensive washing, these samples were incubated with biotinylated goat anti-rabbit secondary antibodies followed by horseradish peroxidase-streptavidin complex. Positive signals were visualized by incubation in peroxidase substrate using diaminobenzidine as the chromogen. Samples were then counterstained in 0.05\% (wt/vol) methyl green (Sigma-Aldrich).

\section{Acknowledgments}

We thank A. Horiuchi, T. Yamaguchi, and Y. Nakazawa for construction of the Jinhua $\times$ Duroc population; M. Nii for construction of the Large White $\times$ Japanese wild boar population; and members of the DNA Marker Project and the Animal Genome Research Program (NIAS/STAFF) for their many years of support. This work was supported by grants to the Animal Genome and DNA Marker projects from the Ministry of Agriculture, Forestry and Fisheries, Japan, and by a grant-in-aid from the Japan Racing Association.

\section{References}

Burke, A.C., Nelson, C.E., Morgan, B.A., and Tabin, C. 1995. Hox genes and the evolution of vertebrate axial morphology. Development 121: $333-346$.

Chen, J.D. and Evans, R.M. 1995. A transcriptional co-repressor that interacts with nuclear hormone receptors. Nature 377: 454-457.

Chen, F., Cooney, A.J., Wang, Y., Law, S.W., and O'Malley, B.W. 1994. Cloning of a novel orphan receptor (GCNF) expressed during germ cell development. Mol. Endocrinol. 8: 1434-1444.

Chung, A.C., Katz, D., Pereira, F.A., Jackson, K.J., DeMayo, F.J., Cooney, A.J., and O'Malley, B.W. 2001. Loss of orphan receptor germ cell nuclear factor function results in ectopic development of the tail bud and a novel posterior truncation. Mol. Cell. Biol. 21: 663-677.

Cordes, R., Schuster-Gossler, K., Serth, K., and Gossler, A. 2004. Specification of vertebral identity is coupled to Notch signalling and the segmentation clock. Development 131: 1221-1233.

David, R., Joos, T.O., and Dreyer, C. 1998. Anteroposterior patterning and organogenesis of Xenopus laevis require a correct dose of germ cell nuclear factor (xGCNF). Mech. Dev. 79: 137-152.

Ding, J., Yang, L., Yan, Y.T., Chen, A., Desai, N., Wynshaw-Boris, A., and Shen, M.M. 1998. Cripto is required for correct orientation of the anterior-posterior axis in the mouse embryo. Nature 395: 702-707.

Dubrulle, J., McGrew, M.J., and Pourquie, O. 2001. FGF signalling controls somite boundary position and regulates segmentation clock control of spatiotemporal Hox gene activity. Cell 106: 219-232.

Fujishima-Kanaya, N., Toki, D., Suzuki, K., Sawazaki, T., Hiraiwa, H., Iida, M., Hayashi, T., Uenishi, H., Wada, Y., Ito, Y., et al. 2003. Development of 50 gene-associated microsatellite markers using BAC clones and the construction of a linkage map of swine chromosome 4. Anim. Genet. 34: 135-141.

Greco, T.L., Takada, S., Newhouse, M.M., McMahon, J.A., McMahon, A.P., and Camper, S.A. 1996. Analysis of the vestigial tail mutation demonstrates that Wnt-3a gene dosage regulates mouse axial development. Genes \& Dev. 10: 313-324.

Haley, C.S., Knott, S.A., and Elsen, J.M. 1994. Mapping quantitative trait loci in crosses between outbred lines using least squares. Genetics 136: $1195-1207$.

Hanson, R.D., Hess, J.L., Yu, B.D., Ernst, P., van Lohuizen, M., Berns, A., van der Lugt, N.M., Shashikant, C.S., Ruddle, F.H., Seto, M., et al. 1999. Mammalian Trithorax and Polycomb group homologues are antagonistic regulators of homeotic development. Proc. Natl. Acad. Sci. 96: 14372-14377.

Hentschke, M., Kurth, I., Borgmeyer, U., and Hubner, C.A. 2006. Germ cell nuclear factor is a repressor of CRIPTO-1 and CRIPTO-3. J. Biol. Chem. 281: 33497-33504.

Horlein, A.J., Naar, A.M., Heinzel, T., Torchia, J., Gloss, B., Kurokawa, R., Ryan, A., Kamei, Y., Soderstrom, M., Glass, C.K., et al. 1995. Ligand-independent repression by the thyroid hormone receptor mediated by a nuclear receptor co-repressor. Nature 377: 397-404.

Hoshino, M., Sone, M., Fukata, M., Kuroda, S., Kaibuchi, K., Nabeshima, Y., and Hama, C. 1999. Identification of the stef gene that encodes a novel guanine nucleotide exchange factor specific for Rac1. J. Biol. Chem. 274: 17837-17844.

Katz, D., Niederberger, C., Slaughter, G.R., and Cooney, A.J. 1997. Characterization of germ cell-specific expression of the orphan nuclear receptor, germ cell nuclear factor. Endocrinology 138: $4364-4372$.

King, J.W.B. and Roberts, R.C. 1960. Carcass length in the bacon pig: Its association with vertebrae numbers and prediction from radiographs of the young pig. Anim. Prod. 2: 59-65.

Lan, Z.J., Chung, A.C., Xu, X., DeMayo, F.J., and Cooney, A.J. 2002. The embryonic function of germ cell nuclear factor is dependent on the DNA binding domain. J. Biol. Chem. 277: 50660-50667. 
Lan, Z.J., Gu, P., Xu, X., and Cooney, A.J. 2003. Expression of the orphan nuclear receptor, germ cell nuclear factor, in mouse gonads and preimplantation embryos. Biol. Reprod. 68: 282-289.

Luo, X., Ikeda, Y., and Parker, K.L. 1994. A cell-specific nuclear receptor is essential for adrenal and gonadal development and sexual differentiation. Cell 77: 481-490.

McPherron, A.C., Lawler, A.M., and Lee, S.-J. 1999. Regulation of anterior/posterior patterning of the axial skeleton by growth/differentiation factor 11. Nat. Genet. 22: 260-264.

Mikawa, S., Shimanuki, S., Morozumi, T., Domukai, M., Shinkai, H., Uchida, Y., Mikawa, A., Miyake, M., Miyake, Y., Hayashi, T. et al. 2004. Comparative analysis and development of microsatellite markers on swine (Sus scrofa) chromosome 1qter. Anim. Genet. 35: $445-450$.

Mikawa, S., Hayashi, T., Nii, M., Shimanuki, S., Morozumi, T., and Awata, T. 2005. Two quantitative trait loci on Sus scrofa chromosomes 1 and 7 affecting the number of vertebrae. J. Anim. Sci. 83: 2247-2254.

Narita, Y. and Kuratani, S. 2005. Evolution of the vertebral formulae in mammals: A perspective on developmental constraints. J. Exp. Zool. 304: 91-106.

Nichols, J., Zevnik, B., Anastassiadis, K., Niwa, H., Klewe-Nebenius, D., Chambers, I., Scholer, H., and Smith, A. 1998. Formation of pluripotent stem cells in the mammalian embryo depends on the POU transcription factor Oct4. Cell 95: 379-391.

Niwa, H., Miyazaki, J., and Smith, A.G. 2000. Quantitative expression of Oct-3/4 defines differentiation, dedifferentiation or self-renewal of ES cells. Nat. Genet. 24: 372-376.

Pilbeam, D. 2004. The anthropoid postcranial axial skeleton: Comments on development, variation, and evolution. J. Exp. Zool. 302: 241-267.

Sato, N., Kondo, M., and Arai, K. 2006. The orphan nuclear receptor GCNF recruits DNA methyltransferase for Oct-3/4 silencing. Biochem. Biophys. Res. Commun. 344: 845-851.

Shen, W.H., Moore, C.C.D., Ikeda, Y., Parker, K.L., and Ingraham, H.A.
1994. Nuclear receptor steroidogenic factor 1 regulates the Mullerian inhibiting substance gene: A link to the sex determination cascade. Cell 77: 651-661.

Suzuki, K., Asakawa, S., Iida, M., Shimanuki, S., Fujishima, N., Hiraiwa H., Murakami, Y., Shimizu, N., and Yasue, H. 2000. Construction and evaluation of a porcine bacterial artificial chromosome library. Anim. Genet. 31: 8-12.

Visscher, P.M., Thompson, R., and Haley, C.S. 1996. Confidence intervals in QTL mapping by bootstrapping. Genetics 143: $1013-1020$.

Wada, Y., Akita, T., Awata, T., Furukawa, T., Sugai, N., Inage, Y., Ishii, K., Ito, Y., Kobayashi, E., Kusumoto, H., et al. 2000. Quantitative trait loci (QTL) analysis in a Meishan $\times$ Göttingen cross population. Anim. Genet. 31: 376-384.

Yan, Z. and Jetten, A.M. 2000. Characterization of the repressor function of the nuclear orphan receptor retinoid receptor-related testis-associated receptor/germ cell nuclear factor. J. Biol. Chem. 275: 35077-35085.

Yan, Z., Kim, Y.S., and Jetten, A.M. 2002. RAP80, a novel nuclear protein that interacts with the retinoid-related testis-associated receptor. J. Biol. Chem. 277: 32379-32388.

Yang, G., Zhang, Y.L., Buchold, G.M., Jetten, A.M., and O'Brien, D.A 2003. Analysis of germ cell nuclear factor transcripts and protein expression during spermatogenesis. Biol. Reprod. 68: 1620-1630.

Yu, B.D., Hess, J.L., Horning, S.E., Brown, G.A., and Korsmeyer, S.J. 1995. Altered Hox expression and segmental identity in Mll-mutant mice. Nature 378: 505-508.

Zhang, Y.L., Akmal, K.M., Tsuruta, J.K., Shang, Q., Hirose, T., Jetten, A.M., Kim, K.H., and O'Brien, D.A. 1998. Expression of germ cell nuclear factor (GCNF/RTR) during spermatogenesis. Mol. Reprod. Dev. 50: $93-102$.

Received October 31, 2006; accepted in revised form February 5, 2007. 


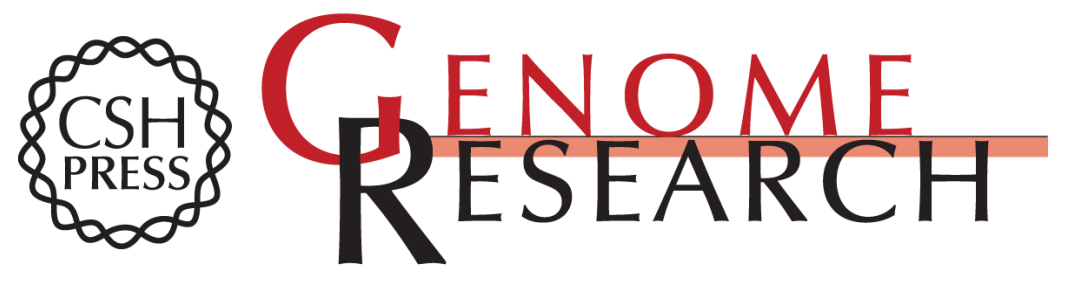

\title{
Fine mapping of a swine quantitative trait locus for number of vertebrae and analysis of an orphan nuclear receptor, germ cell nuclear factor (NR6A1)
}

Satoshi Mikawa, Takeya Morozumi, Shin-Ichi Shimanuki, et al.

Genome Res. 2007 17: 586-593 originally published online April 6, 2007

Access the most recent version at doi:10.1101/gr.6085507

Supplemental http://genome.cshlp.org/content/suppl/2007/04/09/gr.6085507.DC1
Material

References This article cites 37 articles, 12 of which can be accessed free at:

http://genome.cshlp.org/content/17/5/586.full.html\#ref-list-1

\author{
License
}

Email Alerting Receive free email alerts when new articles cite this article - sign up in the box at the Service top right corner of the article or click here.

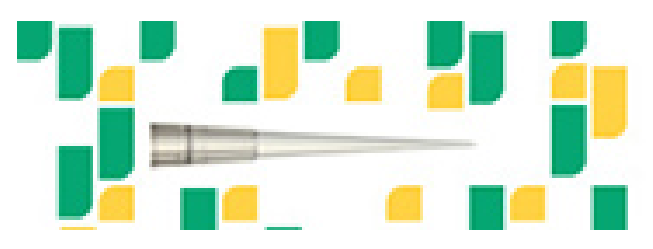

Focused on your science.

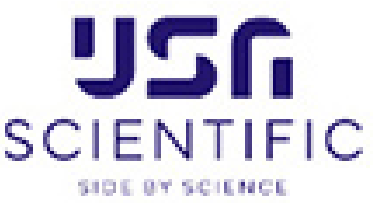

To subscribe to Genome Research go to:

https://genome.cshlp.org/subscriptions 